\title{
The MATROS project: Stability of Uranus and Neptune Trojans. The case of 2001 QR322
}

\author{
F. Marzari ${ }^{1}$, P. Tricarico $^{1}$, and H. Scholl ${ }^{2}$ \\ 1 Dipartimento di Fisica, University of Padova, Via Marzolo 8, 35131 Padova, Italy \\ 2 Observatoire de la Côte d'Azur, BP 4229, 06304 Nice Cedex 4, France
}

Received 17 April 2003 / Accepted 8 August 2003

\begin{abstract}
We present in this paper an analysis of the long term stability of Trojan type orbits of both Uranus and Neptune. Employing the Frequency Map Analysis (hereinafter FMA) we measure the diffusion speed in the phase space for a large sample of Trojan orbits with short numerical integrations. High resolution diffusion maps are derived for different values of initial inclination. These maps outline where the most stable orbits can be found in the Trojan clouds of the two planets.

The orbit of the newly discovered Neptune Trojan 2001 QR322 has been analysed in detail with the FMA method. In the phase space the body is located close to the border of a stable region for low inclination Neptune Trojans. Numerical integrations over 4.5 Gyr of clone orbits generated from the covariance matrix show that only $10 \%$ of the clones escape from the Trojan cloud. The proper frequencies of the Trojan motion computed with the FMA algorithm allow us to to derive a numerical secular theory. From this theory it is possible to locate in the phase space the main secular resonances that can perturb Trojan orbits of the two planets and lead to instability.
\end{abstract}

Key words. solar system: general - celestial mechanics - minor planets, asteroids

\section{Introduction}

The recent discovery of the first Neptune Trojan 2001 QR322 by the Lowell Observatory's Deep Ecliptic Survey team (Buie, Wasserman, and Millis) has triggered additional theoretical and numerical investigations of the stability of Trojan-type orbits of the outer planets. Exploring the long-term stability of 2001 QR322 and of similar bodies, that could be detected in the future around the Lagrangian points of Neptune, is the first step towards a deeper understanding of the origin of Trojantype bodies, and of the formation of their parent planets. 2001 QR322 could be the first member of a consistent primordial population of Neptune Trojans. This finding would have profound implications on the proposed scenarios for the formation of both Uranus and Neptune. If the two planets formed close to their present location on a timescale of the order of $10^{7}$ years (Bryden 2000), local planetesimals could have been trapped as Trojans in this early phase (Marzari et al. 2003). A low or moderate planetary migration would not completely destabilize Trojan orbits according to Gomes (1998) and Fleming \& Hamilton (2000) and we should observe at present this population. On the other hand, it has been proposed by Thommes et al. (2002) that Uranus and Neptune formed in the region between Jupiter and Saturn at the same time as the two giant planets and were then gravitationally scattered outwards. It is critical for this model to evaluate the impact of the scattering phase on the stability of primordial Trojan populations of the two planets

Send offprint requests to: F. Marzari, e-mail: marzari@pd.infn.it and the possibility of a Trojan trapping phase at the end of the fast migration process.

Even the consistency of the potential population of Neptune Trojans could give important clues on how the planet formed and on the timescale of the process. The capture by the mass growth of the planet (Marzari \& Scholl 1998a,b; Fleming \& Hamilton 2000) is effective if the expansion of the libration regions, caused by the increasing gravity field of the planet, is fast. This may not have been the case for Uranus and Neptune if they formed in the outer regions of the solar system. More plausible in this case would be the capture by mutual collisions between planetesimals populating the region surrounding the two planets during their formation (Shoemaker et al. 1989; Marzari et al. 2003). A small population would be expected in this scenario since the trapping by mutual collisions is less efficient than the mass growth mechanism.

The previous speculations on the implications of a population of Trojan asteroids for the formation of Uranus and Neptune are based on the assumption that, once trapped, Trojans can survive over 4.5 Gyr. The stability of tadpole orbits of the two planets was investigated in a systematic way via numerical integrations over 20 Myr by Holman \& Wisdom (1993). They found stable areas for both Uranus and Neptune with some asymmetry L4 vs. L5 possibly due to the geometrical configuration of the planets at the time of selection of the initial conditions. Weissman \& Levison (1997) integrated the orbits of 70 hypothetical Neptune Trojans over $4.5 \mathrm{Gyr}$ and they found that some are indeed stable over the solar 
system age. A more complete survey of the dynamical stability of Uranus and Neptune Trojans was performed by Nesvorny \& Dones (2002) by computing the maximum LCE (Lyapunov Characteristic Exponent). They outline the stability areas in the semimajor axis vs. eccentricity plane and find a higher stability for Neptune Trojans respect to the Uranus ones. The same authors in their paper were urging observes to search for Neptune Trojans due to their stability properties in advance of the discovery of $2001 \mathrm{QR} 322$.

In our paper we adopt a different approach based on the Frequency Map Analysis (hereinafter FMA; Laskar et al. 1993; Laskar 1993a,b; Sidlichovský \& Nesvorný 1997) that allows to estimate the diffusion rate of an orbit in the proper elements space. This method has been previously applied to study dynamical stability of minor bodies in the solar system (Nesvorny \& Ferraz-Mello 1997; Melita \& Brunini 2001; Marzari et al. 2002). With the FMA analysis we can draw diffusion maps for Trojans and determine the regions with higher stability in the proper elements space. Our survey extends also to highly inclined orbits since we selected the initial conditions within different slices in inclination. A natural outcome of the FMA method is the value of the proper frequencies of Trojan orbits as a function of the proper eccentricity and proper libration amplitude. A synthetic theory of the secular behaviour of Uranus and Neptune Trojans is then produced by fitting the proper frequencies of the perihelion longitude, node, and critical argument of all the bodies in our sample. Glimpses at the possible sources of instability related to secular resonances are obtained by comparing the proper frequency of the perihelion longitude and node longitude with the main frequencies of the Solar System.

Once we have identified the regions where the diffusion rate is slower, we can integrate over the solar system age some sample orbits within these regions and explore the possibility that primordial Trojans can have survived till present. We have also studied with both the FMA method and by long term integrations the stability of $2001 \mathrm{QR} 322$ and computed its proper elements in order to locate it within our diffusion portraits.

\section{The numerical model}

The numerical model is extensively described in the previous papers on the MATROS project (Marzari et al. 2002, 2003). Here we briefly summarize the main features of the numerical algorithms:

- The orbits of fictitious Trojans are integrated within a fivebody model including the Sun and the four outer planets. The initial orbital parameters of the planets are taken from the JPL ephemeris. The reference plane is the invariable plane of the solar system.

- The initial conditions for fictitious Trojans are chosen by a preliminary numerical integration over $10^{6}$ years that selects Trojan-type orbits possibly stable over a longer timescale. The orbits are selected within a ring surrounding the orbit of the planet. Only those bodies with the critical argument still librating after $10^{6}$ years are integrated farther in time and analysed with the FMA.
- Different slices in initial inclination $I_{0}$ are considered starting from $I_{0}=0^{\circ}$ and increasing with steps of $10^{\circ}$. Since the work of Holman \& Wisdom (1993) it has been believed that there is an asymmetry in the profile of the stable regions of Neptune Trojans. Their Fig. 1 showing the location of stable orbits over $20 \mathrm{Myr}$ in the semimajor axis vs. initial longitude plane for Neptune Trojans indicates clearly an asymmetry between L4 and L5. L5 Trojans are shifted towards larger initial semimajor axes. While generating the initial conditions we noticed a similar asymmetry related to the number of stable orbits found for the two Lagrangian points. This was simply due a particular initial configuration of the planets at time $t=0$ of the numerical integration. In fact, by shifting the planets $5 \mathrm{Myr}$ ahead in time the asymmetry was reversed. To prevent any bias in our data we always run the code to generate initial conditions twice, once with the planet at $t=0$ and once with the planets at $t=5 \mathrm{Myr}$.

- The main integration of Trojan orbits selected with the preliminary integration extends over 15 Myr for Uranus Trojans and 30 Myr for Neptune Trojans. The output is digitally filtered to remove short periods up to $500 \mathrm{yr}$.

- The FMA algorithm (the numerical code, based on Sidlichovský \& Nesvorný 1997, can be retrieved at the site http://orsa.sourceforge.net as part of the orsa package) is applied to the non-singular variables $h=$ $e \cos (\tilde{\omega})$ and $k=e \sin (\tilde{\omega})$, output of the main integrations. To measure the diffusion speed of each orbit in frequency space, we compute the proper frequency $g$ with the FMA algorithm over running windows of $5 \mathrm{Myr}$ for Uranus and $10 \mathrm{Myr}$ for Neptune. The negative logarithm of the standard deviation $s_{g}$ of $g$ over the windows $\left(\sigma=-\log _{10}\left(s_{g} / g\right)\right)$ is used to monitor the diffusion rate. Larger values of $\sigma$ imply a slower diffusion rate and then higher stability.

- For each orbit the proper eccentricity $e_{\mathrm{p}}$ is computed as the amplitude of proper frequency $g$ in the first window. The proper libration amplitude $D$ is estimated independently as the average of the maximum libration amplitude over short running windows. This algorithm to compute $D$ has proven to be accurate when compared, for Jupiter Trojans, to the values estimated by Milani (1994) (Marzari et al. 2003). The proper frequency $s$ and the proper inclination $I_{\mathrm{p}}$ are computed from the spectral analysis of the $p$ and $q$ variables over the whole timespan of the numerical integration. The period of $s$ is sometimes comparable to the time interval of the integrations.

- Diffusion portraits are produced for each slice of initial inclination $I_{0}$.

- Within the regions with large values of $\sigma$, and hence less chaotic, we pick up starting conditions for long term integrations to estimate dynamical lifetimes over the solar system age.

\section{Diffusion maps for Neptune Trojans}

Over 8000 Orbits of Neptune Trojans have been integrated for $30 \mathrm{Myr}$ and then analysed with the FMA method. In Fig. 1 we show the diffusion maps for different values of the initial 

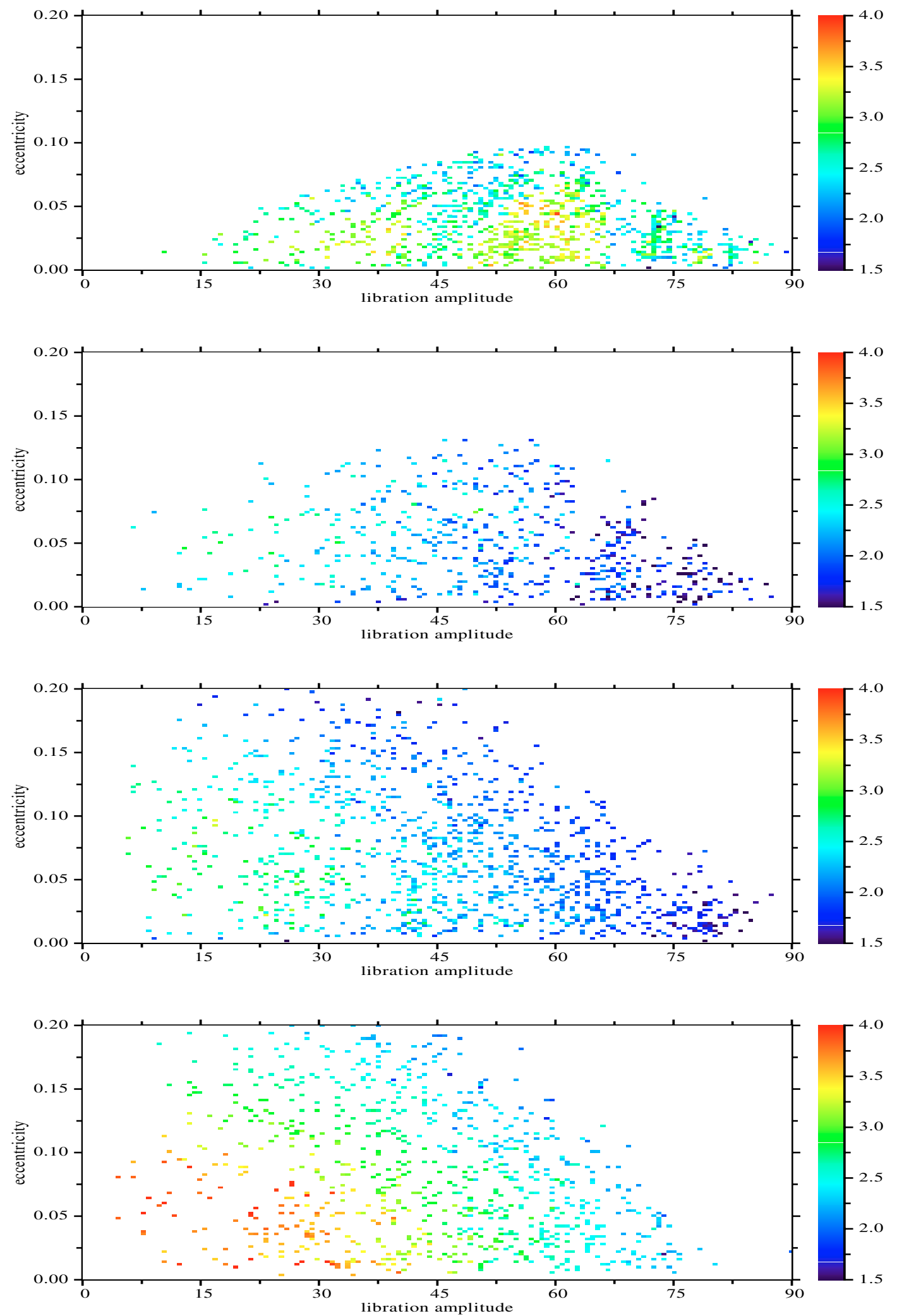

Fig. 1. Diffusion maps of Neptune Trojans for different values of initial inclination $I_{0}$. From top to bottom: $I_{0}=0^{\circ}, I_{0}=10^{\circ}, I_{0}=20^{\circ}$, and $I_{0}=30^{\circ}$.

inclination $I_{0}=0^{\circ}, 10^{\circ}, 20^{\circ}, 30^{\circ}$. There are two high stability regions, one located at low initial inclination $\left(I_{0}=0^{\circ}\right)$, low proper eccentricity $\left(e_{\mathrm{p}}<0.1\right)$, and proper libration amplitude $D$ encompassed between $45^{\circ}$ and $65^{\circ}$. This region is characterized by values of $\sigma$ slightly lower than those characterizing Jupiter Trojans (Marzari et al. 2003) but higher than those of Saturn 
Trojans (Marzari et al. 2002). We expect that Trojans located in this region may survive over the solar system age. This hypothesis is reinforced by the discovery of 2001 QR322 whose proper elements are located within the stable region at $I_{0}=0^{\circ}$. Moreover, the long term integration of clones of 2001 QR322, that will be discussed in the next section, shows that indeed bodies started within the stable area survive for $4.5 \mathrm{Gyr}$. The second stable region is located at $I_{0}=30^{\circ}$, proper eccentricity lower than 0.15 , and libration amplitude $D$ lower than $45^{\circ}$. The orbits in this region are even less chaotic than in the previous stable region at $I_{0}=0^{\circ}$ as their values of $\sigma$ are larger (slower diffusion).

In the diffusion portrait for $I_{0}=0^{\circ}$ there are no stable orbits with low libration amplitude $\left(D<15^{\circ}\right)$. This is similar to the behaviour of Saturn Trojans (Marzari et al. 2002) but opposite to that of Jupiter Trojans. The latter, in fact, at low inclinations are stable for small values of $D$ (Marzari et al. 2003). For low inclination Neptune Trojans and, in particular, for Uranus Trojans, as we will see in Sect. 5, an additional phenomenon work against low libration orbits. Indirect perturbations by the other planets force oscillations of the critical argument for any choice of the starting conditions. When we consider highly inclined Neptune Trojans, the stability geometry progressively changes and low libration orbits become possible and even posses a higher stability. This is clearly demonstrated in Fig. 1 at $I_{0}=30^{\circ}$ where the "red" area, characterized by very low diffusion speed and then high stability, is located at libration amplitudes smaller than $45^{\circ}$. In the diffusion portrait for $I_{0}=20^{\circ}$, orbits with low libration amplitude have instead a moderate diffusion speed. Their values of $\sigma$ are about one order of magnitude larger than the "red" orbits at $I_{0}=30^{\circ}$ suggesting that they are chaotic. However, they may survive as Trojans a few billions of years as in the case of highly inclined Jupiter Trojans (Marzari et al. 2003). The time required to the orbit to increase its libration amplitude until a close encounter with the planet occurs is comparable with the age of the solar system (Marzari et al. 2003). We selected a sample of 15 fictitious Neptune Trojans with $D<20^{\circ}$ and $I_{0}=20^{\circ}$ and we integrated their orbits over 4.5 Gyr. 5 of them (about $30 \%$ ) escape over $4.5 \mathrm{Gyr}$ while the others show chaotic changes of the critical argument to different extents. In Fig. 2 we show the long term behaviour of three Neptune Trojans that belong to the sample. The first case starts with an initial libration amplitude $D=13^{\circ}$ and it escapes from the Trojan cloud after $3.2 \times 10^{9}$ years. While the proper eccentricity $\left(e_{\mathrm{p}}=0.08\right)$ remains constant, $D$ grows chaotically until the body encounters the planet and escapes. The second case with $D=18^{\circ}$ survives over $4.5 \mathrm{Gyr}$, even though its libration amplitude changes chaotically. Its proper eccentricity $e_{\mathrm{p}}$ is smaller than that of the previous case $\left(e_{\mathrm{p}}=0.06\right)$ which possibly delays a close encounter with the planet. The third case of Fig. 2 has an initial $D=9^{\circ}$ and a low proper eccentricity $\left(e_{\mathrm{p}}=0.04\right)$. The libration amplitude steadly grows with time by a small amount but, even in this case, the orbit is stable over the solar system age. From these examples we can infer that Neptune Trojans with initial inclination of about $20^{\circ}$ and low libration amplitude can survive for $4.5 \mathrm{Gyr}$ even if their orbits are chaotic. Their diffusion speed is low enough to grant that most of them

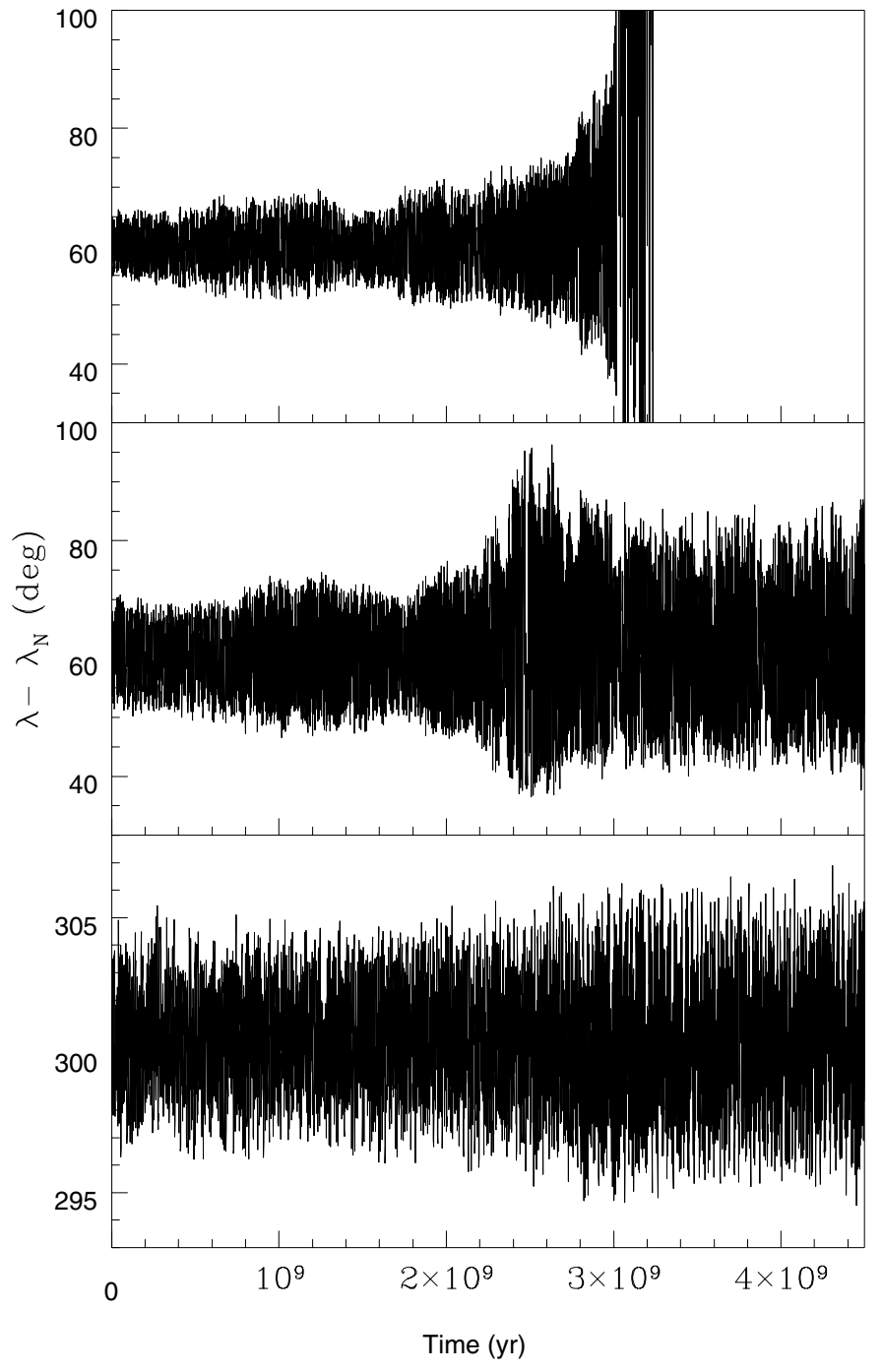

Fig. 2. Time evolution of the critical argument $\lambda-\lambda_{N}$ of 3 Neptune Trojans with initial inclination $I_{0}=20^{\circ}$.

will reach an orbital configuration allowing close encounters with Neptune only later than 4.5 Gyr. Trojan orbits with initial inclination around $I_{0}=30^{\circ}$ and low libration amplitude posses the same stability properties of observed Jupiter Trojans (Marzari et al. 2002, 2003) and a few sample cases have survived over the solar system age without large variations in their orbital elements.

Our results obtained with the FMA method cannot be directly compared to the contour plot of dynamical lifetimes shown in Weissman \& Levison (1997) (Fig. 8, p. 590) since they collect in a single figure orbits with different initial inclinations. For example, the stable area over 4 Gyr they find at low libration amplitude is possibly made of orbits with initial inclinations larger than $10-20^{\circ}$. Nesvorny \& Dones (2002) performed a 4 Gyr survey of Neptune Trojans and they observe as most of the orbits with amplitudes lower than $60-70^{\circ}$ survive confirming the outer limit of the stable areas we outline in Fig. 1. Our results obtained with the FMA extend those of Nesvorny \& Dones (2002) since FMA permits a high resolution and fast coverage of the phase space with short term numerical 


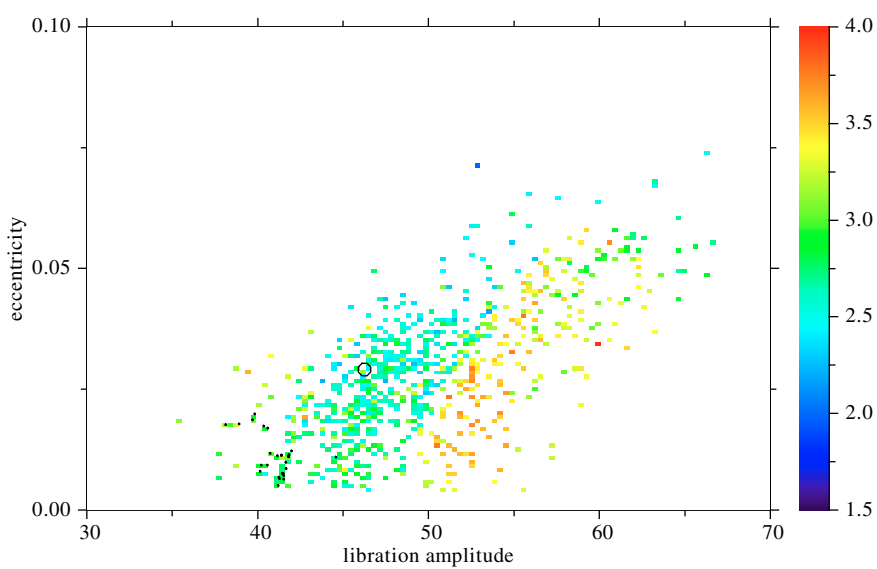

Fig. 3. Diffusion map of clones of the real Neptune Trojan 2001 QR322. We have zoomed in respect to Fig. 1 to gain resolution. The initial conditions have been computed from the covariance matrix. The empty circle is the nominal orbit.

integrations. This has allowed us to obtain an additional resolution in inclination. Moreover, the FMA approach naturally leads to diffusion maps in proper elements which are the preferred elements to explore the secular dynamics of Trojans.

\section{2001 QR322}

The newly discovered Neptune Trojan 2001 QR322 is on a low inclination, almost circular orbit. The cosmogonical implications of its existence are all related to its long term stability. In order to test whether it can be a primordial body, we took the nominal orbit and, from the covariance matrix (hamilton.dm.unipi.it/astdys), we randomly generated 1000 clones. They have been integrated over $30 \mathrm{Myr}$ in order to be analysed with the FMA algorithm and to compute their diffusion speed in the proper element space. In Fig. 3 we show the outcome of the FMA analysis for all the clones (the axis limits in Fig. 3 have been reduced respect to Fig. 1 to increase the resolution). They are located close to the inner border of the stable area identified in Fig. 1 between $45^{\circ}<D<65^{\circ}$. The clones with higher values of $\sigma$ (the "red" dots) have a quasiperiodic behaviour while clones with a higher diffusion speed (the "green-blue" dots) show a chaotic behaviour on a short timescale. The time evolution of the critical argument of two extreme cases is illustrated in Fig. 3. The upper plot show a clone with a high value of $\sigma(\sim 3.7)$ and, as a consequence, a low diffusion speed: its behaviour is regular. In the bottom plot we show the critical argument of a case with a small $\sigma$ value ( 2.6) implying more chaos. The libration amplitude of this clone has significant short term variations over a timescale of $100 \mathrm{Myr}$. We have also integrated for $4.5 \mathrm{Gyr} 70$ clones randomly selected among the 1000, and we find that only $10 \%$ of the clones, those with a higher diffusion speed, escape from the Trojan cloud. These computations show that the dynamically stable region outlined in Fig. 1 with a value of $\sigma$ between 3.2 and 3.8 can possibly harbour Trojan orbits surviving from the early phases of solar system formation. The orbit of 2001 QR322 is close to the edge of this region and may then be primordial. Search for additional Neptune Trojans should focus on this area that, for observational purpose, has to be translated from proper elements into osculating elements.

Brasser et al. (http://www . astro.queensu.ca/ wiegert/ preprints/pp18.html) show that some clones of 2001 QR322, computed changing the last digits of the nominal orbit, are perturbed by the $s-s_{8}$ secular resonance. In Sect. 6 we compute the exact location of this resonance in the phase space with a semiempirical secular theory for Neptune Trojans. Figure 8 shows that the $s-s_{8}$ resonance affects only marginally the cluster of clones we generated from the covariance matrix. Only bodies with libration amplitude around $40-45^{\circ}$ and eccentricity lower than 0.04 have the nodal frequency close to $s_{8}$. These bodies are also marked with a black dot in Fig. 3. We integrated over 4.5 Gyr some of these clones, but they do not show the behaviour described in Brasser et al. The critical argument of the $s-s_{8}$ resonance circulates very slowly but it does not switch from circulation to libration, or invert the circulation trend, in those bodies that escape before $4.5 \mathrm{Gyr}$. The ejection from the Trojan cloud occurs because of a fast increase of the libration amplitude that leads to a close encounter with the planet. The instability source that causes high diffusion speeds (blue-green dots) in all Netpune Trojans close to $D \sim 40^{\circ}$ and with eccentricity up to 0.1 seems to be unrelated to the $s-s_{8}$ secular resonance. The resonance has to be very narrow in the phase space if we did not find it with our sampling procedure. The results of Brasser et al. show that it may affect a few neptune Trojans. However, the present uncertainties in the orbit of 2001 QR322 and the sharp profile of the resonance do not allow strong statements on the influence of this resonance on the real orbit of the Neptune Trojan.

\section{Diffusion maps for Uranus Trojans}

From the diffusion portraits presented in Fig. 5 we can guess that Uranus Trojans are mostly unstable over the solar system age. As for Saturn Trojans (Marzari et al. 2002) and Neptune Trojans for low inclinations, there are no stable low libration amplitude orbits. However, there is a substantial difference between the dynamics of low librating Saturn Trojans and Uranus Trojans. While Saturn Trojans at low values of $D$ become quickly unstable because of resonant perturbations (de La Barre et al. 1996; Marzari et al. 2002; Nesvorny \& Dones 2002), low libration Trojan orbits of Uranus do not exist. Indirect perturbations on the mean longitude of the object from the other planets cumulate and force oscillations of the resonant angle. In Fig. 6 we show an example of such behaviour. A body is started exactly at the location of the L4 Lagrangian point and, notwithstanding that, it librates with an average amplitude of $50^{\circ}$. Even by applying the more general method to search for starting conditions outlined in Sect. 2. we did not find any low libration orbits. This behaviour has important implications when one trys to compare the stability maps drawn in the space of initial $a$ and $e$, as in Holman \& Wisdom (1993) and Nesvorny $\&$ Dones (2002), with our diffusion maps in proper eccentricity and libration amplitude. Orbits that has an initial semimajor axis close to that of the planet do not necessarily map into 
low libration orbits, but may correspond to orbits with large values of $D$. While there is not an easy relation between the initial $d a=a-a_{\mathrm{U}}$ and the libration amplitude of the corresponding orbit, the relation between the oscillations in $a$ and $\lambda-\lambda_{\mathrm{U}}$ (Milani 1993) is instead preserved with good approximation. By inspecting Fig. 5, we conclude that the minimum value for the libration amplitude of Uranus tadpole orbits is approximately encompassed between $15^{\circ}$ and $30^{\circ}$, depending on the inclination. The higher values of $\sigma$, corresponding to the most stable orbits, are found for $I_{0}=0^{\circ}$ around proper eccentricities $e_{\mathrm{p}} \sim 0.05$ and $e_{\mathrm{p}} \sim 0.15$. These appear as "green" areas in the top plot of Fig. 5. The two areas are cut through by the $s_{7}$ secular resonance whose location can be computed from the empirical secular theory derived in Sect. 6. The diffusion speed of the "green" regions is similar to that of the most stable Saturn Trojans (Marzari et al. 2002) whose half-life was found to be about $2.5 \mathrm{Gyr}$. As a consequence, only a few bodies are expected to survive over $4.5 \mathrm{Gyr}$.

In Fig. 5 the diffusion maps for initial inclination $I_{0}=20^{\circ}$ do not appear since we could not find orbits stable at least for 10 Myr. The reason of this high chaoticity is related to secular resonances involving the fundamental frequencies $g_{5}$ and $g_{7}$. Figure 6 , which illustrates the spectrum of the $h$ and $k$ variables, shows clearly that orbits with inclination in between $I_{0}=10^{\circ}$ and $I_{0}=30^{\circ}$ cross the region in the frequency space where both $g_{5}$ and $g_{7}$ are located. Moreover, orbits with inclination $I_{0}=30^{\circ}$ are still strongly perturbed by the $g_{7}$ frequency, whose forced value in eccentricity is comparable to the proper eccentricity of the orbit. The closeby frequency $2 g_{7}-g_{5}$ also contributes to destabilize Uranus Trojans with $I_{0}=30^{\circ}$ and its presence can be seen in Fig. 6 at a frequency $f \sim 1.46 \times 10^{-6} \mathrm{yr}^{-1}$. Additional sources of instability for Uranus Trojans can be three-body resonances, as for Jupiter (Marzari et al. 2003), related to the closeness of Uranus and Neptune to a 2:1 mean motion resonance.

\section{An empirical secular theory for Uranus and Neptune Trojans}

A useful approach to understand the dynamical structure of Uranus and Neptune Trojans is to provide analytical formulas to compute the main frequencies of tadpole orbits as a function of proper elements. With the FMA algorithm we obtain a catalogue of the proper elements and of the corresponding proper frequencies for each of the orbits we have integrated. We have used the same polynomial expression used to fit Jupiter Trojans as in Milani (1994) and in Marzari et al. (2003) to build a secular model for Uranus and Neptune Trojans. We use the following variables in the polynomial expansions:

$$
x=\frac{e_{\mathrm{p}}}{0.15} \quad y=\frac{\sin I_{\mathrm{p}}}{0.6} \quad z=\frac{d a}{0.15 \mathrm{AU}} .
$$

The best fit to the proper frequencies $s, g$, and $f_{\mathrm{L}}$ (libration frequency) of Neptune Trojans yields:

$$
\begin{aligned}
f_{\mathrm{L}}= & 268.77+31.20 x^{2}-24.60 y^{2}-6.30 z^{2}-21.59 y^{4} \\
& +0.48 z^{4}-46.43 x^{2} y^{2}-0.06 x^{2} z^{2}+3.44 y^{2} z^{2}
\end{aligned}
$$

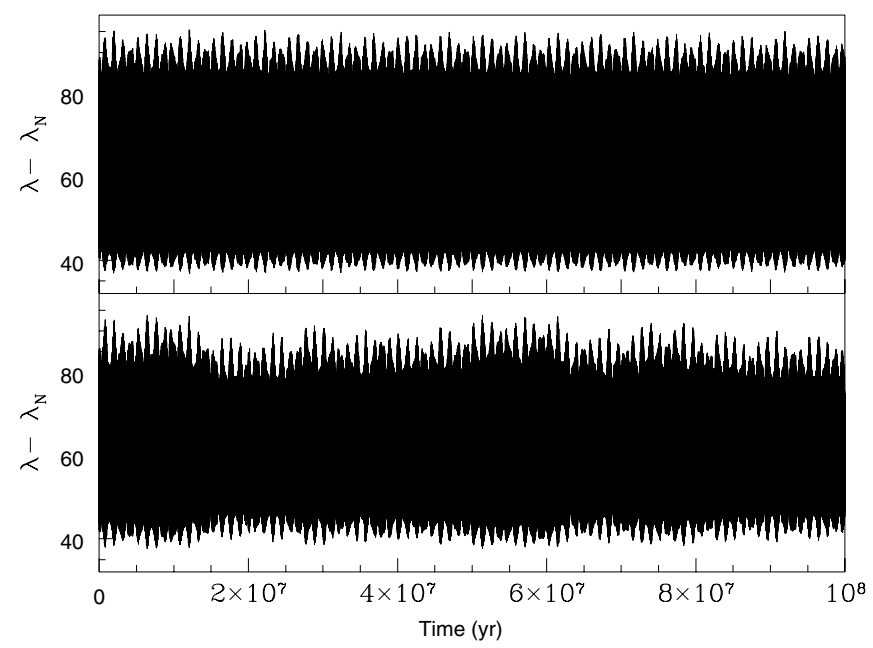

Fig. 4. Evolution with time of the critical argument $\lambda-\lambda_{N}$ of two clones of 2001 QR322. The top plot shows an orbit with a small value of $\sigma$ and hence with a slow diffusion rate. The bottom plot illustrates the behaviour of a clone with a faster diffusion speed and then more chaotic.

$$
\begin{aligned}
s= & -0.66-0.08 x^{2}+0.45 y^{2}-0.03 z^{2}-0.15 y^{4} \\
& -2.35 \times 10^{-4} z^{4}+0.04 x^{2} y^{2} \\
& +5.53 \times 10^{-3} x^{2} z^{2}-1.05 \times 10^{-3} y^{2} z^{2} \\
g= & 2.07-0.01 x^{2}-1.64 y^{2}+0.12 z^{2}+0.70 y^{4}+0.01 z^{4} \\
& +2.59 \times 10^{-3} x^{2} y^{2}-0.05 x^{2} z^{2}-0.12 y^{2} z^{2}
\end{aligned}
$$

The frequencies are expressed in arcsec/yr and the relative error of the coefficients is less than $1 \%$. The value of $d a$ is computed from the libration amplitude $D$ via the equation $d a=\sqrt{(3 \mu)} \cdot a \cdot D$ (Milani 1993) where $\mu$ is the ratio (mass of the planet)/(mass of the planet + mass of the Sun), $a$ is the semimajor axis of the planet in AU, and D is in radians. For Neptune Trojans this equality leads to $d a=D \cdot 0.3738$, while for Uranus $d a=D \cdot 0.2196$. Some of the coefficients in the fit are small, in particular in the expansions of $s$ and $g$. We decided to include them anyway, for sake of precision.

Figure 8 illustrates the location in the Neptune Trojan region of the main secular frequencies, as computed from Eqs. (3) and (4). We consider only those values of inclination used for the diffusion portraits. At low inclinations, the $s_{8}$ secular frequency crosses the area populated by Neptune Trojans. The secular resonance $s-s_{8}$ might then be responsible for the instability of a few clones of 2001 QR322 (see Fig. 4). However, the signature of this secular resonance is not observed in the diffusion map for $I_{0}=0^{\circ}$ while, ad example, the effects of the $s-s_{7}$ secular resonance are clearly marked in the diffusion portrait of Uranus Trojans (see below). This seems to suggest that the perturbations induced by the $s-s_{8}$ resonance are not strong enough to cause a short term instability.

For Uranus Trojans, the fits to the proper frequencies give the following analytical expressions:

$$
\begin{aligned}
f_{L}= & 147.03-8.47 x^{2}-21.47 y^{2}-0.670 z^{2}+3.88 x^{2} y^{2} \\
& +8.07 x^{2} z^{2}+0.16 y^{2} z^{2}-1.15 z^{4}+1.80 y^{4}
\end{aligned}
$$



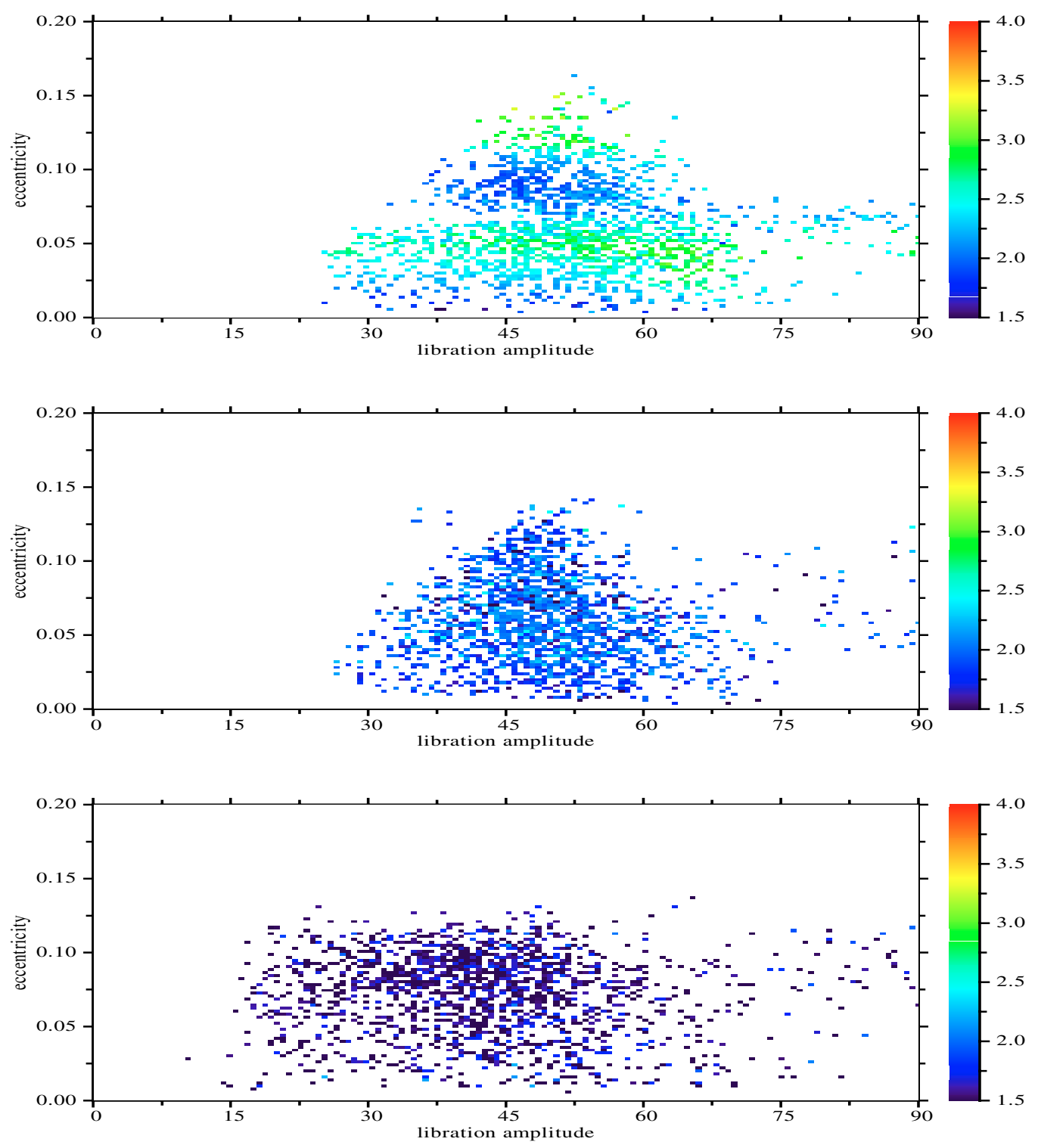

Fig. 5. Diffusion portraits of Uranus Trojans for different values of $I_{0}$. From top to bottom $I_{0}=0^{\circ}, I_{0}=10^{\circ}$, and $I_{0}=30^{\circ}$.

$$
\begin{aligned}
s= & -2.94-0.24 x^{2}+2.32 y^{2}-0.02 z^{2}-1.25 y^{4}+1.28 \\
& \times 10^{-3} z^{4}+0.23 x^{2} y^{2}+0.08 x^{2} z^{2}-6.99 \times 10^{-3} y^{2} z^{2} \\
g= & 5.69-0.24 x^{2}-8.36 y^{2}+0.04 z^{2}+5.43 y^{4}-0.02 z^{4} \\
& -0.34 x^{2} y^{2}-0.29 x^{2} z^{2}+4.90 \times 10^{-3} y^{2} z^{2}
\end{aligned}
$$

Even in this case these numerical fits can be successfully used to locate in the space of proper elements secular resonances with the fundamental frequencies of the Solar System. In Fig. 9 we show the major perturbing secular frequencies for the same values of inclination adopted in the diffusion maps. Particularly noticeable is the secular resonance with $s_{7}$ that cuts the region populated by low inclination Uranus Trojans at $e_{\mathrm{p}} \sim 0.07$. In the diffusion map (Fig. 5) this secular resonance appears to split the relative stable "green" area in two lobes separated by a region of fast diffusion speed.

In Sect. 5 we claimed that Uranus Trojans with initial inclination around $20^{\circ}$ are unstable on a short timescale since they are located close to the two fundamental frequencies $g_{5}$ and $g_{7}$.

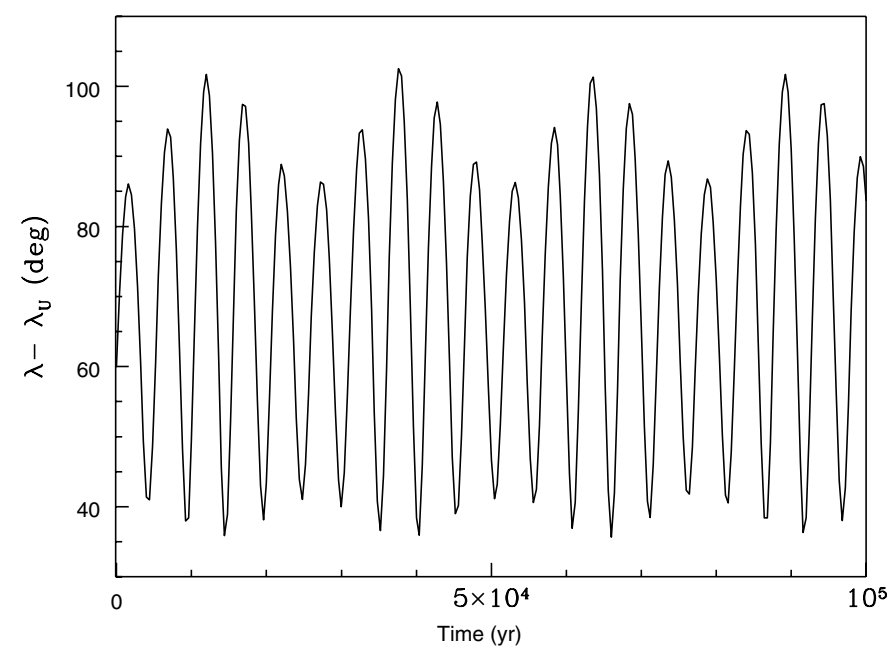

Fig. 6. Evolution of the critical angle of a test Uranus Trojan started with $a=a_{\mathrm{U}}, \lambda-\lambda_{\mathrm{U}}=60^{\circ}$, and $e=0.05 . a_{\mathrm{U}}$ is the semimajor axis of Uranus. 


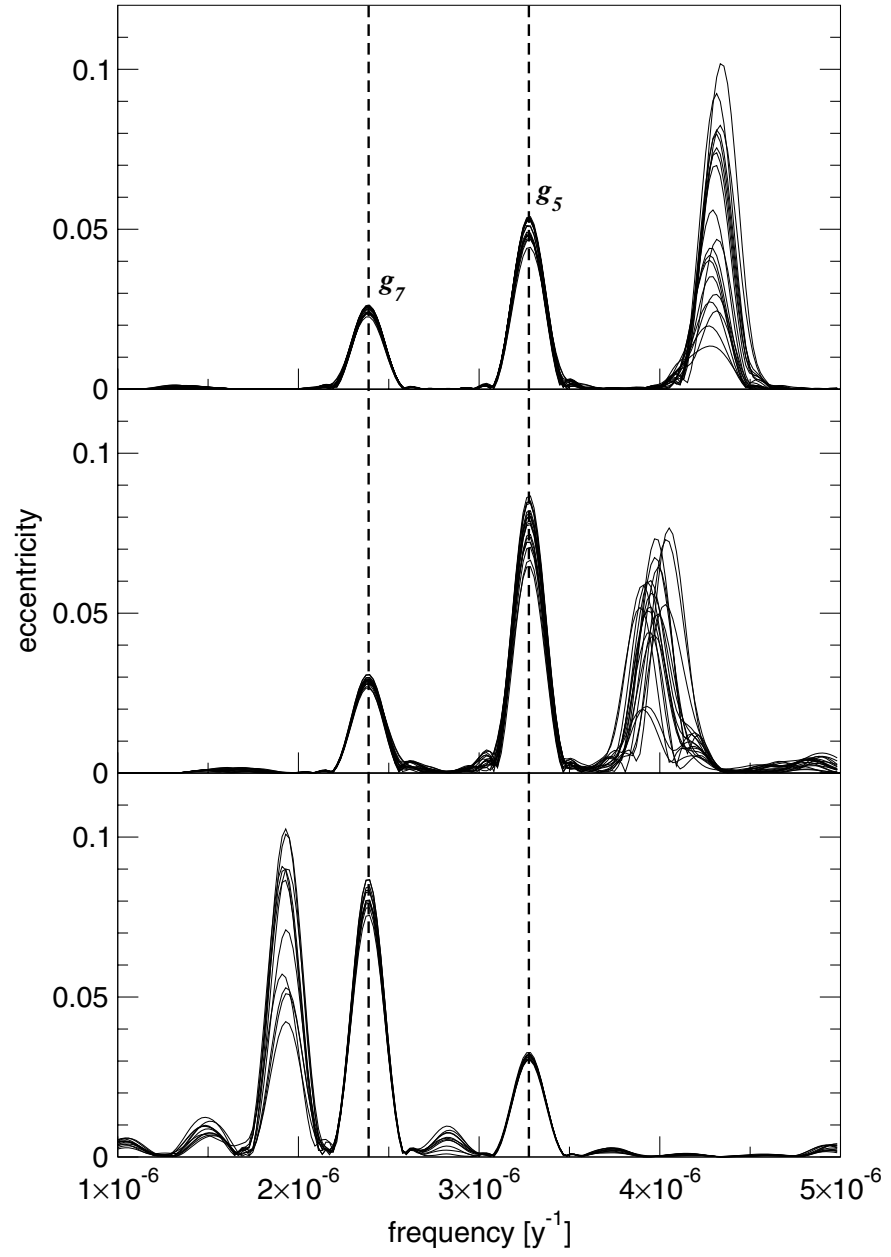

Fig. 7. Power spectra of the $h$ and $k$ variables for Uranus Trojans with different initial inclinations $\left(I_{0}=0,10,30^{\circ}\right)$. When the inclination increases, the proper frequency shifts towards smaller frequencies and it crosses the $g_{7}$ and $g_{5}$ secular resonances. Only for large values of $I_{0}$ the proper frequency moves away from $g_{7}$ and $g_{5}$.

These resonances, however, do not appear in Fig. 9 since in this figure we consider only values of inclination related to the diffusion maps showed in Fig. 5. Since the proper frequency changes very rapidly with inclination for Uranus Trojans (see Eq. (7)), both the $g_{5}$ and $g_{7}$ resonances exit from the $D-e_{\mathrm{p}}$ ranges by increasing the proper inclination. However, by using Eq. (7)) we can determine the range of inclination where Uranus Trojan orbits are perturbed by the two secular resonances $g-g_{5}$ and $g-g_{7}$. In Fig. 10 we show the interval covered by the proper frequency $g$ at different values of inclination for $0.00<e_{\mathrm{p}}<0.15$, and for $15^{\circ}<D<80^{\circ}$. The choice of this region in $D-e_{\mathrm{p}}$ is dictated by the diffusion maps of Fig. 5. The proper frequency $g$ enters the region in between the two secular resonance for $I_{\mathrm{p}} \sim 14^{\circ}$ and exits from it for $I_{\mathrm{p}} \sim 24^{\circ}$. In this interval of inclinations it is difficult to find Trojan orbits stable over a long timescale.

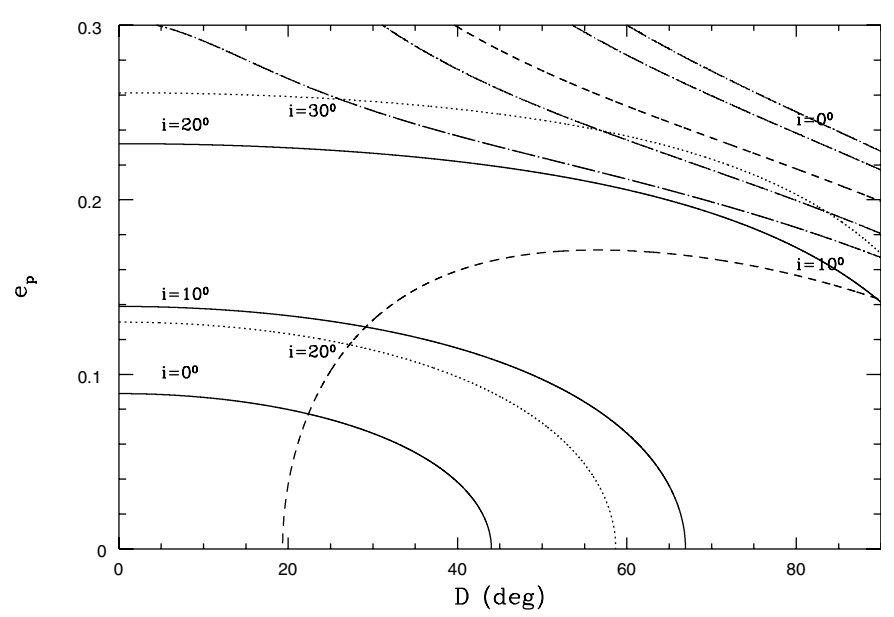

Fig. 8. This figure shows the location of the main secular frequencies that cross the Trojan region of Neptune. The continuous line marks the $s_{8}$ frequency for different proper inclinations, the dotted line the $g_{7}-g_{8}+s_{7}$, and the dashed line the $g_{5}+s_{7}-s_{8}$. The dotted-dashed lines illustrate two additional secular frequencies located at high eccentricity and high libration amplitude: the $2 g_{7}-g_{5}$ and the $g_{5}-g_{7}+g_{8}$.

\section{Conclusions}

The application of the FMA method to Trojan-type orbits of Uranus and Neptune, as presented in this paper, and of Jupiter and Saturn, published in previous papers (Scholl et al. 2002; Marzari et al. 2002, 2003), allows a comparison of the dynamical structure of Trojan regions of the four planets. The diffusion maps obtained by the FMA method yield relative timescales for diffusion that can be converted into absolute timescales by additional numerical integration of selected cases: this is what we have done in a some cases of very slow diffusion. The FMA method may also explain why orbits have a fast diffusion rate. The proper frequencies of Trojans, natural outcome of the FMA analysis, can be compared with the fundamental frequencies of the solar system and possible secular resonances, mixed or higher order resonances, can be easily identified. In case of Jupiter Trojans, we could also detect frequencies related to three-body resonances.

Natural questions concerning the dynamics of Trojans are: do all outer planets have Trojan regions which are stable over the age of the solar system? If yes, where are they located and what is their size. This question can be partially answered by the FMA method. The regions which are stable over the age of the solar system have the highest values of the diffusion coefficient $\sigma$ (and then the slowest diffusion rate). As stated previously, additional numerical integrations over the age of the solar system can confirm whether high $\sigma$ 's mean stability over the age of the solar system. In addition, the FMA method can be used to compare the sizes of stable regions for the four planets and to locate them.

Naively, one would expect to find the most stable regions, those with the longest dynamical lifetimes, very close to the Lagrangian points. For increasing distance from a Lagrangian point, either in the direction of the orbital plane of the parent planet or in the perpendicular direction off the orbital plane, 


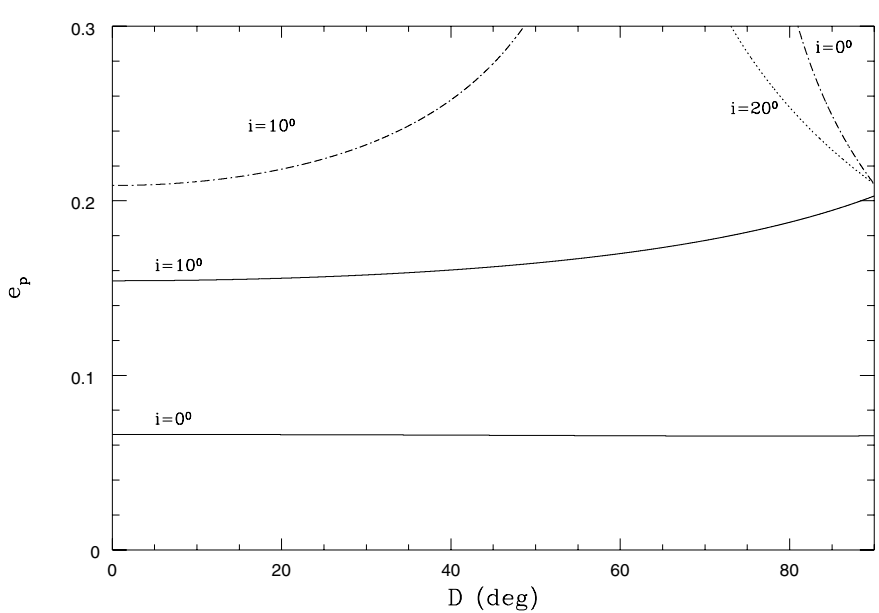

Fig. 9. Location of the main secular frequencies for Uranus Trojans. The continuous line shows the $s_{7}$ frequency for different proper inclinations, the dashed line the $g_{7}$, and the dashed-dotted line the $2 g_{5}-g_{7}$.

intuition suggests that stability should decrease and less bodies should be found. In the FMA "language" this would imply that those Trojan orbits with low inclination and small libration amplitude should posses the largest diffusion coefficients $\sigma$ 's. The diffusion rate would then grow for increasing libration amplitude and increasing inclination. This scenario is only true for Jupiter Trojans, according to our results (Marzari et al. 2003) and to those of Levison et al. (1997) obtained by direct numerical integration while it is only partially true for Trojans of the other three planets Saturn, Uranus and Neptune.

In the case of Saturn Trojans, the diffusion speed is so high at inclinations of $15^{\circ}$ that only a few Trojans survive over 1 Myr. For larger inclinations, the dynamical lifetimes are even shorter. Secular resonances involving nodal frequencies (Marzari et al. 2002) and, possibly, three body resonances (Nesvorny \& Dones 2002), are the major reasons for this fast diffusion. The same behaviour is followed by Uranus Trojans that at inclinations around $20^{\circ}$ are destabilized by secular resonances. Only near $30^{\circ}$ Trojans with somewhat smaller diffusion rate appear while no such cases were found for Saturn Trojans. Secular resonances are then among the main sources of instability for highly inclined Saturn and Uranus Trojans. For Neptune the scenario is different since there is a stable region at low inclination as for the other planets, but near $30^{\circ}$ a large stable area appears with a diffusion speed even lower than that for low inclined tadpole orbits.

A surprising feature of Saturn, Uranus, and Neptune Trojans is the absence of stable orbits at low libration amplitudes. While for Saturn this is due to perturbations that destabilize low libration orbits, for Uranus and Neptune the situation is more complex. Indirect perturbations by the other planets build up in the critical argument and low libration orbits, in particular for Uranus, very often do not exist. The $N$-body problem reveals features that cannot be guessed from the simpler 3-body model.

Finally, by comparing the sizes of Trojan regions with low diffusion rate, corresponding to lifetimes of the order of Gyrs,

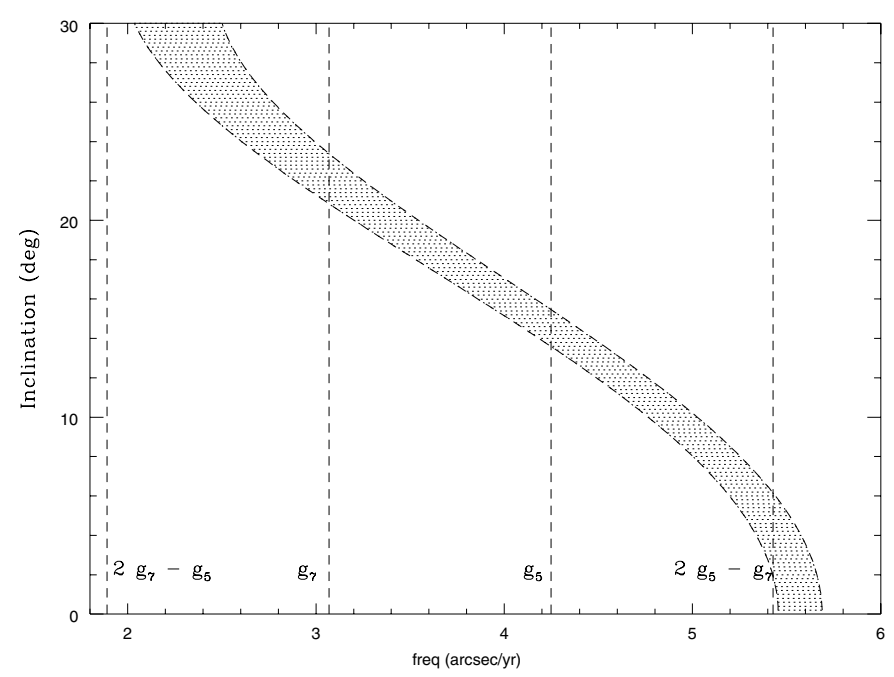

Fig. 10. The shaded area shows the interval of values spanned by the proper frequency $g$ of Uranus Trojans for a given value of proper inclination. For $12^{\circ}<I<24^{\circ}$ the proper frequency $g$ is encompassed between the $g_{7}$ and the $g_{5}$ secular frequencies.

we can say that the largest stability region belongs to Jupiter, followed by Neptune, Saturn and Uranus.

The recently discovered Neptune Trojan 2001 QR322 has a low inclination and a libration amplitude between $45^{\circ}$ and $60^{\circ}$ residing within a region with small diffusion speed. This suggests a lifetime of the order of Gyrs. Numerical integrations of clones of 2001 QR322 over the age of the solar system show that most of them survive. The stable region found at low inclination might then be populated by a consistent primordial swarm of Trojans. The region at low libration amplitude located at $I \sim 30^{\circ}$ is even more stable and could also harbour a primordial population of high inclination Neptune Trojans. As for Jupiter Trojans, the question is how planetesimals initially moving on low inclination orbits could have been excited to such a high inclination.

Acknowledgements. We thank David Nesvorny for useful comments and suggestions.

\section{References}

Bryden, G., Lin, D. N. C., \& Ida, S. 2000, ApJ, 544, 481

de La Barre, C. M., Kaula, W. M., \& Varadi, F. 1996, Icarus, 121, 88

Fleming, H. J., \& Hamilton, D. P. 2000, Icarus, 148, 479

Gomes, R. S. 1998, AJ, 116, 2590

Holman, M. J., \& Wisdom, J. 1993, AJ, 105, 1987

Laskar, J., Froeschlè, C., \& Celletti, A. 1992, Physica D, 56, 253

Laskar, J. 1993, Physica D, 67, 257

Laskar, J. 1993, Cel. Mech. Dyn. Astr., 56, 191

Levison, H., Shoemaker, E. M., \& Shoemaker, C. S. 1997, Nature, 385,42

Marzari, F., Scholl H., Murray, C., \& Lagerkvist, C. 2002, Origin and evolution of Trojan asteroids, in Asteroids III, ed. W. F. Bottke et al. (Tucson: Univ. Of Arizona Press), 725

Marzari, F., Tricarico, P., \& Scholl, H. 2002, ApJ, 579, 905

Marzari, F., \& Scholl, H. 1998, A\&A, 339, 278 
Marzari, F., \& Scholl, H. 1998, Icarus, 131, 41

Marzari, F., Tricarico, P., \& Scholl, H. 2003, Icarus, 162, 453

Marzari, F., Tricarico, P., \& Scholl, H. 2003, MNRAS, submitted

Melita, M. D., \& Brunini, A. 2001, MNRAS, 322, L17

Milani, A. 1994, The dynamics of Trojan asteroids, in Asteroids, Comets, Meteors 1993, 159

Milani, A. 1993, Cel. Mech. Dyn. Astr., 57, 59

Nesvorny, D., \& Dones, L. 2002, Icarus, 160, 271

Nesvorny, D., \& Ferraz-Mello, S. 1997, A\&A, 320, 672

Scholl, H., Tricarico, P., Marzari, F., \& Michel, P. 2002, Results of the MATROS program for Jupiter and Mars Trojans, AAS DPS meeting 34 , abs. n. 02.07
Sidlichovský, M., \& Nesvorný, D. 1997, Cel. Mech. Dyn. Astr., 65, 137

Thommes, E. W., Duncan, M. J., \& Levison, H. F. 2002, AJ, 123, 2862

Shoemaker, E. M., Shoemaker, C. S., \& Wolfe, R. F. 1989, Trojan asteroids: Populations, dynamical structure and origin in L4 and L5 swarms, in Asteroids II, ed. R. Binzel et al. (Tucson: Univ. Of Arizona Press), 487

Weissman, P. R., \& Levison, H. F. 1997, The population of the transneptunian region: The Pluto-charon environment, in Pluto and Charon, ed. S. A. Stern, \& D. J. Tholen (Tucson: Univ. Of Arizona Press), 559 